\title{
Saphenous graft atherosclerosis as assessed by optical coherence tomography data for stenotic and non-stenotic lesions from the OCTOPUS registry
}

\author{
Grzegorz M. Kubiak¹, Elżbieta Pociask², Wojciech Wańha³, Magdalena Dobrolińska3, Paweł Gąsior³, \\ Grzegorz Smolka ${ }^{3}$, Andrzej Ochała ${ }^{3}$, Zbigniew Gąsior ${ }^{4}$, Wojciech Wojakowski ${ }^{3}$ Tomasz Roleder ${ }^{4}$
}

${ }^{1}$ Department of Cardiac Surgery and Transplantology, SMDZ in Zabrze, Medical University of Silesia in Katowice, Silesian Centre for Heart Disease, Zabrze, Poland

${ }^{2}$ Krakow Cardiovascular Research Institute, Jagiellonian University Medical College, Krakow, Poland

${ }^{3} 3^{\text {rd }}$ Division of Cardiology and Structural Heart Diseases, Department of Medical, University of Silesia, Katowice, Poland

${ }^{4}$ Department of Cardiology, School of Health Sciences, Medical University of Silesia, Katowice, Poland

Adv Interv Cardiol 2018; 14, 2 (52): 157-166

DOI: https://doi.org/10.5114/aic.2018.76407

\begin{abstract}
A bstract
Introduction: Coronary artery bypass grafting ( $C A B G)$, although widely used for a long time in diffuse coronary artery disease (CAD), has serious limitations associated with graft aging and its degeneration.

Aim: The relationship between saphenous vein graft (SVG) plaque morphology assessed by optical coherence tomography (OCT) and clinical findings has not been elucidated yet.

Material and methods: We compared the morphology of SVG in stenotic vs. non-stenotic lesions using OCT imaging in 29 patients hospitalized in our center within the OCTOPUS registry.

Results: Stenotic lesions were characterized by higher incidence of thin-cap fibroatheroma (TCFA) (33\% vs. $0 \%, p=0.0048$ ), thrombus ( $28 \%$ vs. $0 \%, p=0.0008)$, lipid-rich plaque (LRP) $(75 \%$ vs. $35 \%, p=0.0013)$ and plaque within the SVG valve $(19 \%$ vs. $0 \%$, $p=0.0114)$ as compared to non-stenotic lesions. Patients with intimal tearing or rupture (ITR) were older (75.8\% vs. 68.9 years, $p=0.047)$ and had lower left ventricular ejection fraction (LVEF) $(32.0 \%$ vs. $49.7 \%, p=0.001)$ and glomerular filtration rate (GFR) ( 36.0 vs. $73.6 \mathrm{ml} / \mathrm{min} / 1.73 \mathrm{~m}^{2}, p=0.010$ ). Patients with calcified lesions vs. those without had lower high-density lipoprotein (HDL) cholesterol (33.2 vs. $44.1 \mathrm{mg} / \mathrm{dl}, p=0.018$ ), similarly to those with ruptured plaque vs. those without ( $28.3 \mathrm{vs} .41 .7 \mathrm{mg} / \mathrm{dl}, p=0.047$ ).

Conclusions: Presence of ITR was associated with advanced age, decreased LVEF and renal insufficiency. Decreased concentration of HDL was associated with higher occurrence of calcified and ruptured plaque.
\end{abstract}

Key words: coronary artery bypass grafting, saphenous vein graft, optical coherence tomography, thin-cap fibroatheroma, coronary artery disease.

\section{Introduction}

Coronary artery bypass grafting (CABG) is widely applied to treat diffuse coronary artery disease [1]. It offers a significant reduction in mortality at 5 -year follow-up compared to medical treatment only $(10.2 \%$ vs. $15.8 \%$; $p=0.0001$ ) [2]. 88-95\% of arterial conduits remain patent at ten or more years after surgery; thus their utilization is a preferred clinical modality. Unfavorably, their use has serious limitations (restrictions in the use of radial artery, mammary artery harvesting may result in sternal dehiscence and/or mediastinitis), particularly in obese and diabetic patients [3-6].
In contrast, only $32-71 \%$ of saphenous vein grafts (SVG) maintain patency at ten or more years [7-12]. As the re-do CABG has two- to four-fold increased mortality, percutaneous coronary intervention $(\mathrm{PCl})$ within the SVG and/or native vessel remains the method of choice in the treatment of these cases $[13,14]$. Percutaneous coronary intervention for SVG is associated with higher incidence of periprocedural myocardial infarction [15]. There exists a paradigm that atherosclerotic plaque localized in the venous conduits consists of friable tissue being prone to release its debris and cause distal embolization during $\mathrm{PCl}[16-19]$.

\section{Corresponding author:}

Grzegorz M. Kubiak MD, Department of Cardiac Surgery and Transplantology, SMDZ in Zabrze, Medical University of Silesia in Katowice, Silesian Centre for Heart Disease, 9 Curie-Skłodowska St, $41-800$ Zabrze, Poland, phone: +48 3237338 57, e-mail: gkubiak@sccs.pl Received: 2.03.2018, accepted: 6.03.2018. 
There is a paucity of data concerning SVG plaque burden and tissue type. The majority of these observations were made before the introduction of optical coherence tomography $(\mathrm{OCT})$ to the clinical setting. Hence, the question arises whether there is a significant difference between the stenotic and non-stenotic regions of the SVG as assessed by OCT imaging.

\section{Aim}

Therefore, the aim of the study was to compare the morphology of SVG in stenotic vs. non-stenotic lesions imaged by OCT, and to present these differences in relation to clinical settings.

\section{Material and methods}

Twenty-nine patients hospitalized in the Upper Silesia Medical Center between June 2013 and March 2016 were included in the OCTOPUS registry $[20,21]$. Each patient gave informed written content, and the study complied with the Declaration of Helsinki and was accepted by the local ethical committee.

Inclusion criteria were: CABG prior to intervention and coronary artery disease with evidence of active ischemia in non-invasive testing or acute coronary disease. Exclusion criteria were: lack of consent or less than 18 years of age or severe valvular insufficiency or contrast allergy or localization of the lesion preventing safe examination or ST-elevated myocardial infarction.

\section{Optical coherence tomography imaging technique}

The lesion was defined as stenotic when it caused $50 \%$ stenosis as assessed. Otherwise, it was recognized as non-stenotic. The non-stenotic segments of the vessel were assigned for further analysis. The St Jude Ilumien Optis Medical system was used for OCT Imaging. The OCT Dragonfly catheter was advanced through a guiding catheter over a 0.014' guidewire into the SVG via the $6 \mathrm{Fr}$ left radial or femoral approach. The OCT probe was positioned $5 \mathrm{~mm}$ distal to the region submitted to analysis. All OCT images were acquired using automatic pullback triggered by the hand injection of contrast flush. All patients were adequately heparinized with the activated clotting time $>300 \mathrm{~s}$.

\section{Optical coherence tomography image analysis}

The OCT image analysis was performed by an independent core laboratory at Krakow Cardiovascular Research Institute (www.KCRI.org). In the case of a conflict of opinions the analyzed frame was excluded from the analysis. The OCT region of interest (ROI) was defined as the lesion length limited by areas without atheroma or neointimal hyperplasia. The OCT analysis scrutinized serial cross-sectional images of the vessel at $1 \mathrm{~mm}$ in- tervals for both stenotic and non-stenotic de novo SVG lesions. Cross-sectional area (CSA), and vessel lumen diameter were measured every $1 \mathrm{~mm}$. The smallest values for both parameters were defined as the minimal lumen diameter (MLD) of the minimal CSA and were assessed for both types of lesions.

The OCT reference lumen area and reference diameter were estimated at the site of the largest CSA within the analyzed SVG for both de novo SVG lesions and non-stenotic lesions. Percentage lumen diameter and area stenosis were defined as the relative decrease in luminal diameter and CSA of the target lesion compared to the reference lumen diameter and CSA.

Tissue was classified as homogeneous for signal-rich regions, lipid for signal-poor regions with diffuse borders and high signal attenuation, calcified for signal-poor regions with sharp edges, and heterogeneous for poor signal regions without signal attenuation. The length of an arc of lipid and calcium that occupied the vessel wall circumference was measured and expressed in degrees $[22,23]$. The maximal lipid arc and calcium arc were measured. The thickness of the fibrous cap that covered the lipid core was measured in the thinnest part of a signal-rich zone that separated the lipid content from the vessel lumen $(\mu \mathrm{m})$. The fibrous cap thickness was the mean value of three measurements. OCT defined thin-cap fibroatheroma (TCFA) as a lipid-rich plaque (LRP) with fibrous cap thickness $<65 \mu \mathrm{m}$. Also, the presence of plaque rupture (PRT), luminal thrombus, intimal tear or rupture (ITR), tissue friability (FRB) and venous valves was noted during the OCT analysis. An intimal tear was defined as a micro-cavity between the SVG lumen and its media, intimal rupture as a micro-cavity of the intima connected with the SVG lumen, tissue friability as a signal-free zone overlaid with signal-rich tissue inside the SVG wall [24]. Offline OCT image analysis was performed using CAAS Intravascular 2.0 (Pie Medical Imaging BV), and results of intraobserver variability for standard protocols were presented previously [25]. See Figure 1 for different types of plaque morphologies.

\section{Statistical analysis}

Distributions of the examined parameters were analyzed using the Shapiro-Wilk test. Categorical variables were expressed as $n$ and percentage. Continuous variables were expressed as the mean \pm standard deviation (SD) or as the median and the $25^{\text {th }}$ and $75^{\text {th }}$ percentiles (interquartile range). Linear variables with normal distribution were compared using Student's $t$-test. Variables with abnormal distribution were compared using the Mann-Whitney $U$ test. Categorical variables of abnormal distribution were compared using the $\chi^{2}$ test with Yates' correction. Differences between the values were considered statistically significant if $p<0.05$. Analyses were 

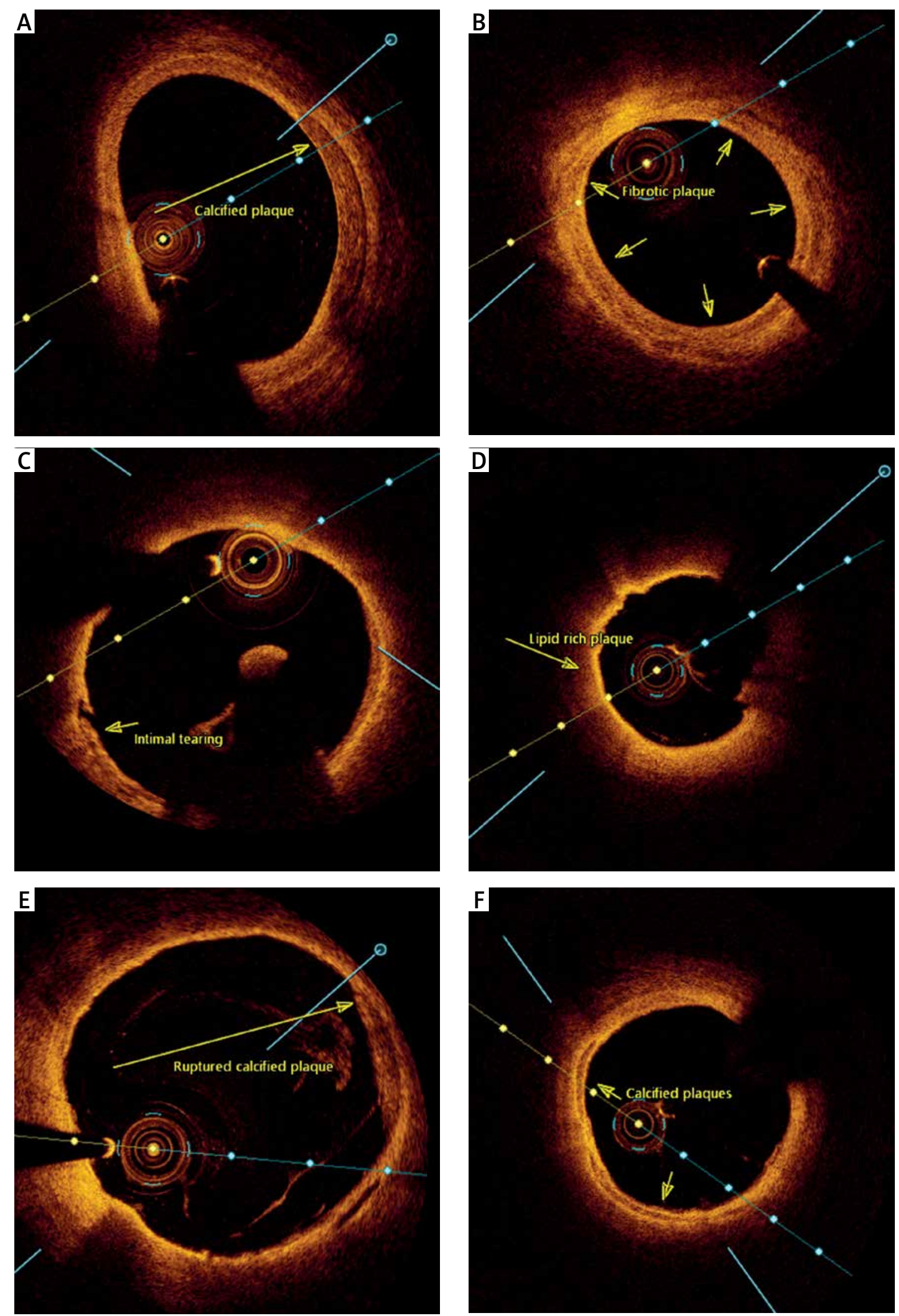

Figure 1. Different types of plaque morphologies. A-F - show different types of plaque morphologies: A - calcified plaque, B - fibrotic plaque, C - intimal tearing, D - lipid-rich plaque, E - ruptured calcified plaque, $\mathbf{F}$ - calcified plaques 
Table I. Patient characteristics $(n=29)$

\begin{tabular}{|c|c|}
\hline Clinical data & Value \\
\hline Age $\pm S D$ & $69.07 \pm 7.56$ \\
\hline Male, $n(\%)$ & $24(83)$ \\
\hline Body mass index, median (IQR) [kg/m²] & $28.5(26-32)$ \\
\hline Non-ST elevated myocardial infarction, $n(\%)$ & $1(3)$ \\
\hline Unstable angina, $n(\%)$ & $10(35)$ \\
\hline Stable angina, $n$ (\%) & $18(62)$ \\
\hline \multicolumn{2}{|l|}{ Risk factors, $n$ (\%): } \\
\hline Hypertension & $26(90)$ \\
\hline Hyperlipidemia & $25(86)$ \\
\hline Diabetes & $13(45)$ \\
\hline Current smoking & $2(7)$ \\
\hline Time from CABG, median (IQR) [months] & $143(100-212)$ \\
\hline \multicolumn{2}{|l|}{ Number of vein conduits, $n(\%)$ : } \\
\hline 1 & $4(14)$ \\
\hline 2 & $18(62)$ \\
\hline 3 & $7(24)$ \\
\hline Arterial conduit (LIMA-LAD) , $n$ (\%) & $26(90)$ \\
\hline \multicolumn{2}{|l|}{ Pharmacological therapy, $n$ (\%): } \\
\hline Aspirin & $28(97)$ \\
\hline Thienopyridine & $2(7)$ \\
\hline$\beta$-Adrenergic antagonist & $25(86)$ \\
\hline Calcium channel antagonist & $4(14)$ \\
\hline $\mathrm{ARB} / \mathrm{ACEI}$ & $20(69)$ \\
\hline Statin & $29(100)$ \\
\hline Other lipid-lowering therapy & $6(21)$ \\
\hline Oral antidiabetics & $5(17)$ \\
\hline Insulin & $2(7)$ \\
\hline \multicolumn{2}{|l|}{ Laboratory results: } \\
\hline Hemoglobin, median (IQR) [mg/dl] & $14.08(12.90-15.22)$ \\
\hline White blood cells, median (IQR) $\left(\times 10^{3} / \mu \mathrm{l}\right)$ & $6.32(5.69-7.24)$ \\
\hline Platelets, median $(\mathrm{IQR})\left(\times 10^{3} / \mu \mathrm{l}\right)$ & $184(161-228)$ \\
\hline Total cholesterol, mean \pm SD [mg/dl] & $162.29 \pm 58.52$ \\
\hline LDL cholesterol, median (IQR) [mg/dl] & $78(68-98)$ \\
\hline HDL cholesterol, median (IQR) [mg/dl] & $41(32-48)$ \\
\hline Triglyceride, median (IQR) [mg/dl] & $132(103-157)$ \\
\hline GFR, median (IQR) [ml/min/1.73 m²] & $71(53-88)$ \\
\hline
\end{tabular}

$S D$ - standard deviation, $I Q R$ - interquartile range, $C A B G$ - coronary artery bypass grafting, LIMA-LAD - left internal mammary artery to left anterior descending artery, ARB - angiotensin II receptor blocker, ACEI - angiotensinconverting-enzyme inhibitor, $L D L$ - low-density lipoprotein, $H D L$ - high-density lipoprotein, GFR - glomerular filtration rate. performed using Statistica 10 with the medical package (StatSoft Inc.).

\section{Results}

\section{Patients' characteristics}

Twenty-nine patients with 32 de novo SVG stenotic and 43 non-stenotic lesions were included in the study. The data for clinical characteristics were depicted on a per patient basis, and the data for plaque morphology were analyzed on a per lesion basis. Percutaneous coronary intervention was performed in 22 of the de novo SVG lesions. The study population consisted of 24 males, mean age $69.07 \pm 7.56$. Mean duration from CABG to the index procedure was 143 (100-212) months. Eighteen $(62 \%)$ patients presented with stable CAD and 11 (38\%) with acute coronary syndrome (ACS). Among ACS patients 10 presented unstable angina symptoms and 1 suffered from non-ST segment elevated myocardial infarction (NSTEMI). For patients' characteristics consisting of clinical data, pharmacological therapy and laboratory findings, see Table I.

\section{Data derived from optical coherence tomography analysis of saphenous vein grafts}

As shown in Table II, stenotic vs. non-stenotic lesions were characterized by a raised plaque burden expressed by lowered MLD (1.88 vs. $2.83 \mathrm{~mm}$ ), increased area stenosis (61.00\% vs. $15.05 \%)$, diameter stenosis (37.33\% vs. $3.0 \%)$ and maximal lipid arc $\left(269^{\circ}\right.$ vs. $\left.97^{\circ}\right) ; p<0.001$ for all. Furthermore, stenotic lesions had a higher incidence of TCFA ( $33 \%$ vs. $0 \%, p=0.0048)$, thrombus ( $28 \%$ vs. $0 \%$, $p=0.0008)$, LRP $(75 \%$ vs. $35 \%, p=0.0013)$, plaque within the SVG valve (19\% vs. $0 \%, p=0.0114)$ and decreased minimal cap thickness (80 vs. $139 \mu \mathrm{m}, p<0.001$ ).

Patients with fibrotic (FIB) tissue were mostly men (26 vs. $9, p=0.004$ ) with higher body surface area (BSA) (2.0 vs. $1.9 \mathrm{~m}^{2}, p=0.014$ ) and increased serum creatinine concentration (1.1 vs. $1.0 \mathrm{mg} / \mathrm{dl}, p=0.028$ ). Moreover, this group of patients was characterized by a positive lipid profile consisting of significantly decreased triglycerides (TG) (116.0 vs. $164.4 \mathrm{mg} / \mathrm{dl}, p=0.013$ ), low-density lipoprotein (LDL) cholesterol (85.1 vs. 101.2, $p=0.05$ ) and elevated high-density lipoprotein (HDL) cholesterol (43.2 vs. $34.8, p=0.06$ ), although neither of the last two $p$-values reached statistical significance. Patients with fibrotic tissue were less frequently current smokers (0 vs. $25 \%$, $p=0.029$ ). On the other hand, patients diagnosed with LRP had higher concentration of platelets (231.9 vs. $\left.182.3 \times 10^{3} / \mu \mathrm{l}, p=0.008\right)$ and were smokers ( $27 \%$ vs. $0 \%$, $p=0.020)$. Data are presented in Table III.

As presented in Table IV, patients with calcified lesions (CAL) had decreased HDL cholesterol (33.2 vs. $44.1 \mathrm{mg} / \mathrm{dl}$, $p=0.018$ ), similarly to those with ruptured plaque (PRT) (28.3 vs. $41.7 \mathrm{mg} / \mathrm{dl}, p=0.047$ ). 
Patients with intimal tearing or rupture (ITR) were older (75.8 vs. 68.9 years, $p=0.047$ ), had significantly impaired systolic function with reduced left ventricular ejection fraction (LVEF) (32.0\% vs. 49.7\%, $p=0.001)$, decreased GFR (36.0 vs. $73.6 \mathrm{ml} / \mathrm{min} / 1.73 \mathrm{~m}^{2}, p=0.010$ ) and total cholesterol (TCH) (93.4 vs. $161.3 \mathrm{mg} / \mathrm{dl}, p=$ 0.033). Patients with diagnosed ITR had raised cardiac troponin concentrations both before and after the procedure (0.6 vs. $0.2 \mathrm{ng} / \mathrm{l}, p=0.05$ for both) of borderline significance. Data are presented in Table V.

\section{Discussion}

According to our best knowledge, there is a lack of systematic comparison between stenotic vs. non-stenotic lesions assessed by OCT; thus, we encountered serious difficulties in addressing the issue in the previously published papers. Our observations concerning stenotic lesions are in line with the work of Davlouros et al. [24] with the exception that the ACS in our group of patients occurred in the minority of cases (11 ACS vs. 18 patients with stable angina), hence TCFA, PRT and ITR were considerably less frequent. What might be a novelty in the current research is that only TCFA, LRP, thrombus and plaque within the valve had a higher incidence rate in stenotic lesions compared to non-stenotic ones. In contrast, presence of PRT, ITR and FRB did not differ significantly. Adlam et al. [26] evaluated sixteen SVGs in asymptomatic patients 3 years after cardiac surgery and reported that the rates of TCFA and thrombus were $37.5 \%$ and $25 \%$ respectively. These data are consistent with our results - incidence of TCFA and thrombus were 33\% and $28 \%$ respectively. Considering the significant imbalance between the time from CABG in Adlam's (3 years) and our (12 years) group of patients, it is tempting to speculate that the thrombus and TCFA formation accelerates in a non-linear way and a major impact on its occurrence is exerted by the quality of conduit tissue and periprocedural surgical conditions. Burgmaier et al. reported the relationship between plaque vulnerability and the left ventricle dilatation assessed by cardiac magnetic resonance

Table II. Comparison of stenotic vs. non-stenotic lesions in saphenous vein grafts (SVG)

\begin{tabular}{|c|c|c|c|}
\hline Parameter & Stenotic lesions $(n=32)$ & Non-stenotic lesions $(n=43)$ & $P$-value \\
\hline Region of interest [mm] & $12.45 \pm 4.99$ & $10.71 \pm 3.85$ & 0.25 \\
\hline Reference lumen CSA [mm²] & 7.41 (IQR: 4.38-9.38) & 7.56 (IQR: 5.60-8.70) & 0.43 \\
\hline Reference mean lumen diameter [mm] & $3.03 \pm 0.73$ & $3.06 \pm 0.46$ & 0.49 \\
\hline Minimal lesion lumen CSA, median (IQR) [mm²] & $2.71(1.34-4.19)$ & NA & NA \\
\hline Minimal lumen diameter [mm] & $1.88 \pm 0.65$ & $2.83 \pm 0.45$ & $<0.001$ \\
\hline Area stenosis, median (IQR) (\%) & $61.00(42.72-77.63)$ & $15.05(13.0-17.0)$ & $<0.001$ \\
\hline Diameter stenosis (\%) & $37.33 \pm 17.25$ & $3.0 \pm 4.82$ & $<0.001$ \\
\hline Minimal cap thickness, median (IQR) [ $\mu \mathrm{m}]$ & $80(60-101)$ & $139(125-155)$ & $<0.001$ \\
\hline Maximal lipid arc, median (IQR) [] & $269(163-317)$ & $97(75-120)$ & $<0.001$ \\
\hline Maximal calcification $\operatorname{arc}\left[{ }^{\circ}\right]$ & $86.89 \pm 54.19$ & $112 \pm 51.6$ & 0.11 \\
\hline Plaque calcification, $n$ (\%) & $14(44)$ & $16(37)$ & 0.74 \\
\hline Thin-cap fibroatheroma, $n$ (\%) & $7(33)$ & $0(0)$ & 0.0048 \\
\hline Thrombus, $n$ (\%) & $9(28)$ & $0(0)$ & 0.0008 \\
\hline Heterogeneous tissue, $n$ (\%) & $2(6)$ & $4(9)$ & 0.96 \\
\hline Plaque rupture, $n$ (\%) & $4(12.5)$ & $4(9)$ & 0.95 \\
\hline Lipid-rich plaque, $n$ (\%) & $24(75)$ & $15(35)$ & 0.0013 \\
\hline Dissection, $n(\%)$ & $1(3)$ & $0(0)$ & 0.88 \\
\hline Intimal tearing, $n(\%)$ & $2(6)$ & $2(5)$ & 0.83 \\
\hline Intimal rupture, $n(\%)$ & $2(6)$ & $3(7)$ & 0.73 \\
\hline Tissue friability, $n$ (\%) & $6(19)$ & $2(5)$ & 0.11 \\
\hline Plaque within the SVG valve, $n(\%)$ & $6(19)$ & $0(0)$ & 0.0114 \\
\hline
\end{tabular}


imaging (CMR) in patients with type two diabetes [27]. We observed significant deterioration in the left ventricle (LV) systolic function in patients diagnosed with ITR (LVEF was $32.0 \%$ vs. $49.7 \%, p=0.001$ ). Moreover, this group of patients exhibited impaired renal function (GFR $36.0 \mathrm{vs.}$ $73.6 \mathrm{ml} / \mathrm{min} / 1.73 \mathrm{~m}^{2}, p=0.010$ ). These data are in line with the previous results of Burgmaier et al., although some important differences should be addressed. First of all, we assessed the patients in a real-world setting; hence LVEF evaluation was performed by the use of ultrasound imaging and the penetration of $\mathrm{HF}$ is considerable $(21 \%$ of patients with LVEF $\leq 35 \%)$. Secondly, despite

Table III. Clinical and imaging findings depending on tissue type according to OCT imaging

\begin{tabular}{|c|c|c|c|c|c|c|}
\hline Parameter & $\begin{array}{c}\text { FIB-0 } \\
(n=16) \\
\text { Mean } \pm \text { SD }\end{array}$ & $\begin{array}{c}\text { FIB-1 } \\
(n=27) \\
\text { Mean } \pm \text { SD }\end{array}$ & $P$-value & $\begin{array}{c}\text { LRP-0 } \\
(n=28) \\
\text { Mean } \pm \text { SD }\end{array}$ & $\begin{array}{c}\text { LRP-1 } \\
(n=15) \\
\text { Mean } \pm \text { SD }\end{array}$ & $P$-value \\
\hline EEM volume [mm] & $123.4 \pm 53.3$ & $124.3 \pm 57.7$ & 0.96 & $115.1 \pm 55.8$ & $140.6 \pm 52.7$ & 0.15 \\
\hline Lumen volume [mm] & $92.9 \pm 40.4$ & $90.9 \pm 44.8$ & 0.71 & $85.2 \pm 43.2$ & $103.7 \pm 40.5$ & 0.09 \\
\hline Min. av. lumen diameter [mm] & $3.1 \pm 0.6$ & $3.0 \pm 0.4$ & 0.56 & $3.0 \pm 0.4$ & $3.1 \pm 0.5$ & 0.40 \\
\hline Min. lumen area $\left[\mathrm{mm}^{2}\right]$ & $7.8 \pm 2.8$ & $7.4 \pm 2.1$ & 0.95 & $7.3 \pm 2.1$ & $8.0 \pm 2.7$ & 0.62 \\
\hline Min. lumen diameter [mm] & $2.9 \pm 0.6$ & $2.8 \pm 0.4$ & 0.45 & $2.8 \pm 0.4$ & $2.9 \pm 0.5$ & 0.20 \\
\hline Plaque volume [mm] & $30.4 \pm 14.2$ & $33.3 \pm 14.5$ & 0.58 & $29.8 \pm 14.0$ & $36.8 \pm 14.2$ & 0.13 \\
\hline Stenosis EEM [\%] & $14.5 \pm 2.5$ & $15.4 \pm 2.3$ & 0.26 & $15.1 \pm 2.3$ & $15.0 \pm 2.7$ & 0.93 \\
\hline Stenosis length [mm] & $10.5 \pm 3.9$ & $10.8 \pm 3.9$ & 0.76 & $10.1 \pm 3.6$ & $11.8 \pm 4.1$ & 0.17 \\
\hline Stenosis reference (\%) & $3.1 \pm 6.5$ & $2.9 \pm 3.6$ & 0.19 & $2.3 \pm 3.6$ & $4.3 \pm 6.5$ & 0.42 \\
\hline Total lumen perimeter $\left[\mathrm{mm}^{2}\right]$ & $109.8 \pm 41.1$ & $111.5 \pm 43.8$ & 0.90 & $103.6 \pm 41.6$ & $124.5 \pm 41.6$ & 0.13 \\
\hline Age [years] & $70.4 \pm 5.5$ & $69.3 \pm 8.4$ & 0.65 & $68.7 \pm 6.4$ & $71.5 \pm 8.7$ & 0.25 \\
\hline Body surface area $\left[\mathrm{m}^{2}\right]$ & $1.9 \pm 0.2$ & $2.0 \pm 0.1$ & 0.014 & $2.0 \pm 0.2$ & $2.0 \pm 0.2$ & 0.68 \\
\hline Body mass index $\left[\mathrm{kg} / \mathrm{m}^{2}\right]$ & $28.0 \pm 2.8$ & $29.6 \pm 3.4$ & 0.16 & $28.9 \pm 3.8$ & $29.0 \pm 2.3$ & 0.98 \\
\hline LVEF (\%) & $49.9 \pm 8.9$ & $46.3 \pm 11.6$ & 0.36 & $46.7 \pm 11.5$ & $49.3 \pm 9.2$ & 0.64 \\
\hline Troponin before [ng/l] & $0.4 \pm 0.5$ & $0.2 \pm 0.4$ & 0.22 & $0.2 \pm 0.4$ & $0.4 \pm 0.5$ & 0.29 \\
\hline Troponin after [ng/l] & $0.4 \pm 0.5$ & $0.2 \pm 0.4$ & 0.22 & $0.2 \pm 0.4$ & $0.4 \pm 0.5$ & 0.29 \\
\hline $\mathrm{HGB}[\mathrm{mg} / \mathrm{dl}]$ & $15.0 \pm 7.3$ & $15.9 \pm 7.1$ & 0.71 & $15.9 \pm 7.1$ & $15.0 \pm 7.1$ & 0.69 \\
\hline WBC $\left[\times 10^{3} / \mu \mathrm{l}\right]$ & $7.1 \pm 1.8$ & $6.9 \pm 1.6$ & 0.54 & $6.9 \pm 1.4$ & $7.1 \pm 2.0$ & 0.97 \\
\hline $\mathrm{PLT}\left[\times 10^{3} / \mu \mathrm{l}\right]$ & $221.1 \pm 65.8$ & $189.7 \pm 40.9$ & 0.19 & $182.3 \pm 31.9$ & $231.9 \pm 65.5$ & 0.008 \\
\hline $\mathrm{TCH}[\mathrm{mg} / \mathrm{dl}]$ & $168.6 \pm 44.4$ & $143.3 \pm 52.2$ & 0.08 & $147.7 \pm 52.2$ & $160.4 \pm 47.9$ & 0.76 \\
\hline $\mathrm{TG}[\mathrm{mg} / \mathrm{dl}]$ & $164.4 \pm 33.1$ & $116.0 \pm 61.0$ & 0.013 & $134.8 \pm 60.4$ & $131.2 \pm 53.3$ & 0.86 \\
\hline $\mathrm{LDL}[\mathrm{mg} / \mathrm{dl}]$ & $101.2 \pm 37.2$ & $85.1 \pm 34.3$ & 0.05 & $88.7 \pm 33.0$ & $94.5 \pm 41.3$ & 0.67 \\
\hline $\mathrm{HDL}[\mathrm{mg} / \mathrm{dl}]$ & $34.8 \pm 9.1$ & $43.2 \pm 14.7$ & 0.06 & $40.5 \pm 14.7$ & $39.8 \pm 11.5$ & 0.81 \\
\hline Creatinine [mg/dl] & $1.0 \pm 0.4$ & $1.1 \pm 0.4$ & 0.028 & $1.1 \pm 0.3$ & $1.0 \pm 0.3$ & 0.13 \\
\hline GFR $\left[\mathrm{ml} / \mathrm{min} / 1.73 \mathrm{~m}^{2}\right]$ & $72.4 \pm 17.9$ & $67.3 \pm 24.7$ & 0.77 & $66.8 \pm 24.6$ & $72.9 \pm 18.7$ & 0.50 \\
\hline Male, $n(\%)$ & $9(56)$ & $26(96)$ & 0.004 & $25(89)$ & $10(67)$ & 0.16 \\
\hline Diabetes, $n(\%)$ & $10(63)$ & $12(44)$ & 0.41 & $14(50)$ & $8(53)$ & 0.91 \\
\hline Hypertension, n (\%) & $16(100)$ & $21(78)$ & 0.12 & $22(79)$ & $15(100)$ & 0.14 \\
\hline Current smoking, $n(\%)$ & $4(25)$ & $0(0)$ & 0.029 & $0(0)$ & $4(27)$ & 0.020 \\
\hline
\end{tabular}

FIB-O/1 - fibrotic tissue absent/present, LRP-O/1 - lipid-rich plaque absent/present, EEM - external elastic membrane, min. - minimal, av. - average, LVEF - left ventricular ejection fraction, HGB - hemoglobin, WBC - white blood cells, PLT - platelets, TG - triglyceride, TCH - total cholesterol, LDL - low-density lipoprotein cholesterol, HDL - high-density lipoprotein cholesterol, GFR - glomerular filtration rate; insignificant p-values were rounded up to two decimal places, significant $p$-values were rounded up to three decimal places. 
the fact that patients with diabetes are prone to glucose fluctuations which are associated with vulnerable plaque formation [28], PRT prevalence in the dilated LV group of patients was higher but without statistical significance (22.7\% vs. $8.5 \%, p=0.083)$. Notably, although many efforts aiming to improve long-term efficacy of SVG have been made throughout the years, interesting theoretical assumptions have not necessarily had a positive impact on clinical practice [29]. Last but not least, to date nothing is known about the relationship between clinical characteristics and SVG plaque morphology assessed by OCT in patients previously submitted to CABG. Therefore

Table IV. Clinical and imaging findings depending on tissue type according to OCT imaging

\begin{tabular}{|c|c|c|c|c|c|c|}
\hline Parameter & $\begin{array}{c}\text { CAL-0 } \\
(n=27) \\
\text { Mean } \pm \text { SD }\end{array}$ & $\begin{array}{c}\text { CAL-1 } \\
(n=16) \\
\text { Mean } \pm \text { SD }\end{array}$ & $P$-value & $\begin{array}{c}\text { PRT-0 } \\
(n=39) \\
\text { Mean } \pm \text { SD }\end{array}$ & $\begin{array}{c}\text { PRT-1 } \\
(n=4) \\
\text { Mean } \pm \text { SD }\end{array}$ & $P$-value \\
\hline EEM volume [mm] & $126.9 \pm 57.5$ & $119.0 \pm 53.4$ & 0.66 & $125.7 \pm 57.5$ & $107.2 \pm 28.2$ & 0.53 \\
\hline Lumen volume [mm] & $93.0 \pm 43.5$ & $89.5 \pm 42.6$ & 0.94 & $92.5 \pm 44.2$ & $83.5 \pm 26.1$ & 0.90 \\
\hline Min. av. lumen diameter [mm] & $3.0 \pm 0.5$ & $3.2 \pm 0.4$ & 0.18 & $3.0 \pm 0.4$ & $3.3 \pm 0.6$ & 0.28 \\
\hline Min. lumen area $\left[\mathrm{mm}^{2}\right]$ & $7.3 \pm 2.3$ & $8.1 \pm 2.3$ & 0.30 & $7.4 \pm 2.2$ & $8.8 \pm 3.2$ & 0.42 \\
\hline Min. lumen diameter [mm] & $2.8 \pm 0.4$ & $2.9 \pm 0.5$ & 0.30 & $2.8 \pm 0.4$ & $3.0 \pm 0.7$ & 0.42 \\
\hline Plaque volume [mm] & $33.9 \pm 15.0$ & $29.4 \pm 13.0$ & 0.41 & $33.1 \pm 14.7$ & $23.6 \pm 2.3$ & 0.17 \\
\hline Stenosis EEM [\%] & $15.3 \pm 2.3$ & $14.6 \pm 2.6$ & 0.32 & $15.1 \pm 2.4$ & $14.5 \pm 2.9$ & 0.64 \\
\hline Stenosis length [mm] & $11.3 \pm 3.8$ & $9.7 \pm 3.9$ & 0.18 & $11.0 \pm 3.9$ & $7.6 \pm 1.0$ & 0.10 \\
\hline Stenosis reference (\%) & $3.9 \pm 5.7$ & $1.6 \pm 2.4$ & 0.19 & $3.2 \pm 5.0$ & $0.8 \pm 1.5$ & 0.25 \\
\hline Total lumen perimeter $\left[\mathrm{mm}^{2}\right]$ & $114.4 \pm 42.0$ & $104.9 \pm 43.5$ & 0.48 & $113.1 \pm 43.6$ & $89.1 \pm 15.7$ & 0.28 \\
\hline Age [years] & $68.3 \pm 8.6$ & $71.9 \pm 4.1$ & 0.12 & $69.1 \pm 7.4$ & $75.8 \pm 2.5$ & 0.08 \\
\hline Body surface area $\left[\mathrm{m}^{2}\right]$ & $2.0 \pm 0.2$ & $2.0 \pm 0.2$ & 0.76 & $2.0 \pm 0.2$ & $1.9 \pm 0.1$ & 0.20 \\
\hline Body mass index $\left[\mathrm{kg} / \mathrm{m}^{2}\right]$ & $29.6 \pm 3.1$ & $27.9 \pm 3.2$ & 0.13 & $29.2 \pm 3.2$ & $26.7 \pm 1.5$ & 0.20 \\
\hline LVEF (\%) & $48.4 \pm 10.2$ & $46.3 \pm 11.6$ & 0.47 & $47.8 \pm 10.7$ & $45.8 \pm 12.1$ & 0.59 \\
\hline Troponin before [ng/l] & $0.2 \pm 0.4$ & $0.3 \pm 0.5$ & 0.50 & $0.3 \pm 0.4$ & $0.0 \pm 0.0$ & 0.41 \\
\hline Troponin after [ng/l] & $0.2 \pm 0.4$ & $0.3 \pm 0.5$ & 0.50 & $0.3 \pm 0.4$ & $0.0 \pm 0.0$ & 0.41 \\
\hline $\mathrm{HGB}[\mathrm{mg} / \mathrm{dl}]$ & $16.6 \pm 8.5$ & $13.5 \pm 1.2$ & 0.18 & $15.8 \pm 7.4$ & $13.6 \pm 1.1$ & 0.56 \\
\hline WBC $\left[\times 10^{3} / \mu l\right]$ & $6.6 \pm 1.2$ & $7.6 \pm 2.2$ & 0.19 & $6.9 \pm 1.5$ & $7.5 \pm 2.6$ & 0.88 \\
\hline $\mathrm{PLT}\left[\times 10^{3} / \mu \mathrm{l}\right]$ & $202.4 \pm 42.0$ & $196.6 \pm 69.4$ & 0.13 & $198.4 \pm 48.1$ & $218.8 \pm 89.7$ & 0.91 \\
\hline $\mathrm{TCH}[\mathrm{mg} / \mathrm{dl}]$ & $164.6 \pm 45.3$ & $129.2 \pm 53.2$ & 0.21 & $153.4 \pm 52.9$ & $141.8 \pm 23.8$ & 0.57 \\
\hline TG [mg/dl] & $132.9 \pm 64.6$ & $134.5 \pm 43.4$ & 0.94 & $130.2 \pm 57.4$ & $159.8 \pm 55.9$ & 0.34 \\
\hline $\mathrm{LDL}[\mathrm{mg} / \mathrm{dl}]$ & $94.2 \pm 42.2$ & $84.3 \pm 18.4$ & 0.90 & $91.8 \pm 37.1$ & $82.3 \pm 21.3$ & 0.86 \\
\hline $\mathrm{HDL}[\mathrm{mg} / \mathrm{dl}]$ & $44.1 \pm 13.3$ & $33.2 \pm 11.0$ & 0.018 & $41.7 \pm 13.3$ & $28.3 \pm 9.1$ & 0.047 \\
\hline Creatinine [mg/dl] & $1.0 \pm 0.3$ & $1.1 \pm 0.4$ & 0.87 & $1.0 \pm 0.3$ & $1.2 \pm 0.6$ & 0.89 \\
\hline GFR $\left[\mathrm{ml} / \mathrm{min} / 1.73 \mathrm{~m}^{2}\right]$ & $71.9 \pm 17.4$ & $63.4 \pm 30.0$ & 0.44 & $69.4 \pm 22.8$ & $64.8 \pm 24.1$ & 0.60 \\
\hline Male, $n(\%)$ & $22(81)$ & $13(81)$ & 0.70 & $32(82)$ & $3(75)$ & 0.74 \\
\hline Diabetes, $n(\%)$ & $13(48)$ & $9(56)$ & 0.84 & $21(54)$ & $1(25)$ & 0.57 \\
\hline Hypertension, $n$ (\%) & $21(78)$ & $16(100)$ & 0.16 & $33(85)$ & $4(100)$ & 0.93 \\
\hline Current smoking, $n(\%)$ & $3(11)$ & $1(6)$ & 0.99 & $4(10)$ & $0(0)$ & 0.82 \\
\hline
\end{tabular}

CAL-O/1 - calcified lesion absent/present, PRT-O/1 - plaque rupture absent/present, EEM - external elastic membrane, min. - minimal, av. - average, LVEF - left ventricular ejection fraction, $H G B$ - hemoglobin, WBC - white blood cells, PLT - platelets, TG - triglyceride, TCH - total cholesterol, $L D L$ - low-density lipoprotein cholesterol, HDL - high-density lipoprotein cholesterol, GFR - glomerular filtration rate; insignificant p-values were rounded up to two decimal places, significant p-values were rounded up to three decimal places. 
we suggest initiating a randomized control trial of SVGs after cardiac surgery to address the issue of OCT-derived plaque morphology with respect to hypothetical clinical benefit in this therapeutically demanding group of patients.

This is a preliminary study that enrolled a relatively small number of patients. As it was performed in one center, although the researchers did not interfere with the management process, there exists a possibility of selection bias. Despite the fact that it was recently widely discussed and considered insignificant, since OCT is an invasive procedure there exists a theoretical possibility of iatrogenic damage of the vessel wall which might have influenced the results.

Table V. Clinical and imaging findings depending on tissue type according to OCT imaging

\begin{tabular}{|c|c|c|c|c|c|c|}
\hline Parameter & $\begin{array}{c}\text { ITR-0 } \\
(n=38) \\
\text { Mean } \pm \text { SD }\end{array}$ & $\begin{array}{c}\text { ITR-1 } \\
(n=5) \\
\text { Mean } \pm \text { SD }\end{array}$ & $P$-value & $\begin{array}{c}\text { FRB-0 } \\
(n=41) \\
\text { Mean } \pm \text { SD }\end{array}$ & $\begin{array}{c}\text { FRB-1 } \\
(n=2) \\
\text { Mean } \pm \text { SD }\end{array}$ & $P$-value \\
\hline EEM volume $[\mathrm{mm}]$ & $125.7 \pm 54.1$ & $111.1 \pm 70.6$ & 0.59 & $126.2 \pm 55.8$ & $77.1 \pm 10.6$ & 0.23 \\
\hline Lumen volume [mm] & $92.9 \pm 42.0$ & $82.5 \pm 52.0$ & 0.52 & $93.3 \pm 43.1$ & $59.1 \pm 9.8$ & 0.12 \\
\hline Min. av. lumen diameter [mm] & $3.0 \pm 0.5$ & $3.2 \pm 0.2$ & 0.47 & $3.1 \pm 0.5$ & $3.0 \pm 0.6$ & 0.74 \\
\hline Min. lumen area $\left[\mathrm{mm}^{2}\right]$ & $7.5 \pm 2.5$ & $8.2 \pm 0.8$ & 0.23 & $7.6 \pm 2.3$ & $7.0 \pm 3.1$ & 0.89 \\
\hline Min. lumen diameter [mm] & $2.8 \pm 0.5$ & $2.9 \pm 0.3$ & 0.86 & $2.8 \pm 0.4$ & $2.7 \pm 0.6$ & 0.69 \\
\hline Plaque volume [mm] & $32.8 \pm 13.9$ & $28.5 \pm 18.8$ & 0.36 & $33.0 \pm 14.3$ & $17.9 \pm 0.8$ & 0.12 \\
\hline Stenosis EEM [\%] & $15.1 \pm 2.5$ & $14.4 \pm 1.1$ & 0.53 & $15.1 \pm 2.4$ & $13.5 \pm 0.7$ & 0.36 \\
\hline Stenosis length [mm] & $10.9 \pm 3.6$ & $9.0 \pm 5.8$ & 0.30 & $10.9 \pm 3.8$ & $7.7 \pm 3.5$ & 0.26 \\
\hline Stenosis reference (\%) & $3.3 \pm 5.0$ & $0.6 \pm 1.3$ & 0.14 & $3.0 \pm 4.9$ & $2.5 \pm 3.5$ & 1.00 \\
\hline Total lumen perimeter $\left[\mathrm{mm}^{2}\right]$ & $112.7 \pm 39.9$ & $97.2 \pm 61.7$ & 0.45 & $112.7 \pm 42.5$ & $74.0 \pm 11.6$ & 0.21 \\
\hline Age [years] & $68.9 \pm 7.2$ & $75.8 \pm 5.7$ & 0.047 & $69.5 \pm 7.5$ & $74.5 \pm 2.1$ & 0.35 \\
\hline Body surface area $\left[\mathrm{m}^{2}\right]$ & $2.0 \pm 0.2$ & $2.1 \pm 0.1$ & 0.09 & $2.0 \pm 0.2$ & $1.8 \pm 0.1$ & 0.25 \\
\hline Body mass index $\left[\mathrm{kg} / \mathrm{m}^{2}\right]$ & $29.0 \pm 3.4$ & $28.4 \pm 2.2$ & 0.69 & $29.0 \pm 3.2$ & $27.5 \pm 3.5$ & 0.52 \\
\hline LVEF (\%) & $49.7 \pm 9.4$ & $32.0 \pm 6.7$ & 0.001 & $47.5 \pm 10.7$ & $50.0 \pm 14.1$ & 0.62 \\
\hline Troponin before [ng/l] & $0.2 \pm 0.4$ & $0.6 \pm 0.5$ & 0.05 & $0.2 \pm 0.4$ & $1.0 \pm 0.0$ & 1.00 \\
\hline Troponin after [ng/l] & $0.2 \pm 0.4$ & $0.6 \pm 0.5$ & 0.05 & $0.2 \pm 0.4$ & $1.0 \pm 0.0$ & 1.00 \\
\hline $\mathrm{HGB}[\mathrm{mg} / \mathrm{dll}]$ & $15.7 \pm 7.5$ & $14.7 \pm 1.6$ & 0.77 & $15.7 \pm 7.2$ & $12.8 \pm 1.3$ & 0.58 \\
\hline $\mathrm{WBC}\left[\times 10^{3} / \mu \mathrm{l}\right]$ & $6.9 \pm 1.6$ & $7.3 \pm 1.7$ & 0.69 & $7.0 \pm 1.7$ & $6.7 \pm 0.0$ & 0.57 \\
\hline PLT $\left[\times 10^{3} / \mu \mathrm{ll}\right]$ & $201.6 \pm 55.3$ & $192.2 \pm 18.4$ & 0.84 & $201.1 \pm 52.9$ & $186.5 \pm 48.8$ & 0.61 \\
\hline $\mathrm{TCH}[\mathrm{mg} / \mathrm{dll}]$ & $161.3 \pm 41.6$ & $93.4 \pm 67.4$ & 0.033 & $151.2 \pm 51.7$ & $169.0 \pm 8.5$ & 0.27 \\
\hline TG [mg/dl] & $140.4 \pm 55.2$ & $90.6 \pm 55.5$ & 0.07 & $131.6 \pm 58.2$ & $165.5 \pm 21.9$ & 0.42 \\
\hline $\mathrm{LDL}[\mathrm{mg} / \mathrm{dl}]$ & $93.1 \pm 37.6$ & $75.4 \pm 12.1$ & 0.27 & $90.4 \pm 36.7$ & $96.0 \pm 4.2$ & 0.33 \\
\hline $\mathrm{HDL}[\mathrm{mg} / \mathrm{dl}]$ & $40.5 \pm 13.0$ & $38.8 \pm 18.2$ & 0.76 & $40.3 \pm 13.8$ & $40.0 \pm 8.5$ & 0.89 \\
\hline Creatinine $[\mathrm{mg} / \mathrm{dl}]$ & $1.0 \pm 0.3$ & $1.2 \pm 0.2$ & 0.16 & $1.1 \pm 0.3$ & $0.8 \pm 0.0$ & 0.31 \\
\hline GFR $\left[\mathrm{ml} / \mathrm{min} / 1.73 \mathrm{~m}^{2}\right]$ & $73.6 \pm 17.4$ & $36.0 \pm 29.8$ & 0.010 & $68.9 \pm 23.2$ & $69.0 \pm 4.2$ & 0.64 \\
\hline Male, $n(\%)$ & $31(82)$ & $4(80)$ & 0.60 & $35(85)$ & $0(0)$ & 0.036 \\
\hline Diabetes, $n$ (\%) & $19(50)$ & $3(60)$ & 0.96 & $20(49)$ & $2(100)$ & 0.49 \\
\hline Hypertension, $n$ (\%) & $32(84)$ & $5(100)$ & 0.79 & $35(85)$ & $2(100)$ & 0.64 \\
\hline Current smoking, $n(\%)$ & $3(8)$ & $1(20)$ & 0.95 & $3(7)$ & $1(50)$ & 0.43 \\
\hline
\end{tabular}

ITR-O/1 - intimal tearing or rupture absent/present, FRB-O/1 - tissue friability absent/present, EEM - external elastic membrane, min. - minimal, av. - average, LVEF left ventricular ejection fraction, HGB - hemoglobin, WBC - white blood cells, PLT - platelets, TG -triglyceride, TCH - total cholesterol, LDL - low-density lipoprotein cholesterol, HDL - high-density lipoprotein cholesterol, GFR - glomerular filtration rate; insignificant p-values were rounded up to two decimal places, significant $p$-values were rounded up to three decimal places. 


\section{Conclusions}

Stenotic lesions of the SVG had a higher incidence of LRP, TCFA, thrombus, and plaque within the valve compared to non-stenotic ones. Presence of ITR was associated with advanced age, deteriorated systolic function of the left ventricle and renal insufficiency. Decreased concentration of HDL was associated with higher occurrence of calcified and ruptured plaque.

\section{Acknowledgments}

This work was supported by European Union structural funds (Innovative Economy Operational Program POIG.01.01.02-00-109/09-00) and statutory funds of the Medical University of Silesia.

\section{Conflict of interest}

The authors declare no conflict of interest.

\section{References}

1. Authors/Task Force members; Windecker S, Kolh P, Alfonso F, et al. 2014 ESC/EACTS Guidelines on Myocardial Revascularization: The Task Force on Myocardial Revascularization of the European Society of Cardiology (ESC) and the European Association for Cardio-Thoracic Surgery (EACTS) Developed with the Special Contribution of the European Association of Percutaneous Cardiovascular Interventions (EAPCI). Eur Heart J 2014; 35: 2541-619.

2. Yusuf S, Zucker D, Peduzzi P, et al. Effect of coronary artery bypass graft surgery on survival: overview of 10-year results from randomised trials by the coronary artery bypass graft surgery Trialists Collaboration. Lancet 1994; 344: 563-70.

3. Hemo E, Mohr R, Uretzky G, et al. Long-term outcomes of patients with diabetes receiving bilateral internal thoracic artery grafts. J Thorac Cardiovasc Surg 2013; 146: 586-92.

4. Taggart DP, Lees B, Gray A, et al.; Investigators ART. Protocol for the Arterial Revascularisation Trial (ART). A randomised trial to compare survival following bilateral vs. single internal mammary grafting in coronary revascularisation [ISRCTN46552265]. Trials 2006; 7: 7.

5. Elmistekawy EM, Gawad N, Bourke M, et al. Is bilateral internal thoracic artery use safe in the elderly? J Card Surg 2012; 27: 1-5.

6. Toumpoulis IK, Theakos N, Dunning J. Does bilateral internal thoracic artery harvest increase the risk of mediastinitis? Interact Cardiovasc Thorac Surg 2007; 6: 787-91.

7. Hattler B, Messenger JC, Shroyer AL, et al. Veterans Affairs Randomized On/Off Bypass (ROOBY) Study Group. Off-pump coronary artery bypass surgery is associated with worse arterial and saphenous vein graft patency and less effective revascularization: results from the Veterans Affairs Randomized On/Off Bypass (ROOBY) Trial. Circulation 2012; 125: 2827-35.

8. Alexander JH, Hafley G, Harrington RA, et al. PREVENT IV Investigators. Efficacy and safety of edifoligide, an E2F transcription factor decoy, for prevention of vein graft failure following coronary artery bypass graft surgery: PREVENT IV: a randomized controlled trial. JAMA 2005; 294: 2446-54.

9. Tatoulis J, Buxton BF, Fuller JA. The right internal thoracic artery: the forgotten conduit--5,766 patients and 991 angiograms. Ann Thorac Surg 2011; 92: 9-15.
10. Barner HB, Bailey M, Guthrie TJ, et al. Radial artery free and T graft patency as coronary artery bypass conduit over a 15-year period. Circulation 2012; 126 (11 Suppl 1): S140-4.

11. Achouh P, Boutekadjirt R, Toledano D, et al. Long-term (5- to 20year) patency of the radial artery for coronary bypass grafting. J Thorac Cardiovasc Surg 2010; 140: 73-9.

12. Sabik JF, Blackstone EH, Houghtaling PL, et al. Is reoperation still a risk factor in coronary artery bypass surgery? Ann Thorac Surg 2005; 80: 1719-27.

13. Yap CH, Sposato L, Akowuah E, et al. Contemporary results show repeat coronary artery bypass grafting remains a risk factor for operative mortality. Ann Thorac Surg 2009; 87: 1386-91.

14. Morrison DA, Sethi G, Sacks J, et al. Investigators of the Department of Veterans Affairs Cooperative Study \#385, Angina With Extremely Serious Operative Mortality Evaluation. Percutaneous coronary intervention versus repeat bypass surgery for patients with medically refractory myocardial ischemia: AWESOME randomized trial and registry experience with post-CABG patients. J Am Coll Cardiol 2002; 40: 1951-4.

15. Coolong A, Baim DS, Kuntz RE, et al. Saphenous vein graft stenting and major adverse cardiac events: a predictive model derived from a pooled analysis of 3958 patients. Circulation 2008; 117: 790-7.

16. Abbo KM, Dooris M, Glazier S, et al. Features and outcome of no-reflow after percutaneous coronary intervention. Am J Cardiol 1995; 75: 778-82.

17. Waksman R, Douglas JS, Scott NA, et al. Distal embolization is common after directional atherectomy in coronary arteries and saphenous vein grafts. Am Heart J 1995; 129: 430-5.

18. Baim DS, Carrozza JP. Understanding the "no-reflow" problem. Cathet Cardiovasc Diagn 1996; 39: 7-8.

19. Piana RN, Paik GY, Moscucci M, et al. Incidence and treatment of "no-reflow" after percutaneous coronary intervention. Circulation 1994; 89: 2514-8.

20. Roleder T, Wanha W, Smolka G, et al. Bioresorbable vascular scaffolds in saphenous vein grafts (data from OCTOPUS Registry). Postep Kardiol Inter 2015; 11: 323-6.

21. Roleder T, Pociask E, Wańha W, et al. Optical coherence tomography of de novo lesions and in-stent restenosis in coronary saphenous vein grafts (OCTOPUS Study). Circ J 2016; 80: 1804-11.

22. Yabushita H, Bouma BE, Houser SL, et al. Characterization of human atherosclerosis by optical coherence tomography. Circulation 2002; 106: 1640-5.

23. Kim JS, Afari ME, Ha J, et al. Neointimal patterns obtained by optical coherence tomography correlate with specific histological components and neointimal proliferation in a swine model of restenosis. Eur Heart J Cardiovasc Imaging 2014; 15: 292-8.

24. Davlouros P, Damelou A, Karantalis V, et al. Evaluation of culprit saphenous vein graft lesions with optical coherence tomography in patients with acute coronary syndromes. JACC CardiovasC Interv 2011; 4: 683-93.

25. Kochman J, Tomaniak M, Kołtowski $Ł$, et al. A 12-month angiographic and optical coherence tomography follow-up after bioresorbable vascular scaffold implantation in patients with ST-segment elevation myocardial infarction. Catheter Cardiovasc Interv 2015; 86: E180-9.

26. Adlam D, Antoniades C, Lee R, et al. OCT characteristics of saphenous vein graft atherosclerosis. JACC Cardiovasc Imaging 2011; 4: 807-9.

27. Burgmaier $M$, Frick $M$, Liberman $A$, et al. Plaque vulnerability of coronary artery lesions is related to left ventricular dilatation 
as determined by optical coherence tomography and cardiac magnetic resonance imaging in patients with type 2 diabetes. Cardiovasc Diabetol 2013; 12: 102.

28. Kuroda M, Shinke T, Otake H, et al. Effects of daily glucose fluctuations on the healing response to everolimus-eluting stent implantation as assessed using continuous glucose monitoring and optical coherence tomography. Cardiovasc Diabetol 2016; $15 ; 79$.

29. Węglarz P, Krejca M, Trusz-Gluza M, et al. Neointima development in externally stented saphenous vein grafts. Adv Interv Cardiol 2016; 12: 334-9. 